\title{
A Counter-rotating Bulge in the Sb Galaxy NGC 7331
}

\author{
F. Prada ${ }^{1}$ \\ C.M. Gutiérrez ${ }^{1,2}$ \\ R.F. Peletier ${ }^{3,1}$ \\ and \\ C. D. McKeith ${ }^{4}$ \\ ${ }^{1}$ Instituto de Astrofísica de Canarias, 38200 La Laguna, Tenerife, Spain \\ ${ }^{2}$ University of Manchester, Nuffield Radio Astronomy Laboratories, Jodrell Bank, \\ Macclesfield, Cheshire, SK11 9DL, UK \\ ${ }^{3}$ Kapteyn Laboratorium, P.O. Box 800, 9700 AV Groningen, The Netherlands \\ ${ }^{4}$ Department of Pure and Applied Physics, Queen's University of Belfast, Belfast \\ BT7 1NN, UK
}

\begin{abstract}
We have found that the bulge of the large, nearby Sb galaxy NGC 7331 rotates retrograde to its disk. Analysis of spectra in the region of the near-IR Ca II triplet along the major axis shows that, in the radial range between $5^{\prime \prime}$ and $\sim 20^{\prime \prime}$, the line of sight velocity distribution of the absorption lines is has two distinct peaks, and can be decomposed into a fast-rotating component with $\mathrm{v} / \sigma>3$, and a slower rotating, retrograde component with $\mathrm{v} / \sigma \sim 1-1.5$. The radial surface brightness profile of the counter-rotating component follows that of the bulge, obtained from a 2-dimensional bulge-disk decomposition of a near-infrared $K$-band image, while the fast rotating component follows the disk. At the radius where the disk starts to dominate the isophotes change from being considerably boxy to very disky.

Although a number of spiral galaxies have been found that contain cold, couterrotating disk, this is the first galaxy known to have a boxy, probably triaxial, fairly warm, counter-rotating component, which is dominating in the central regions. If it is a bar seen end-on, this bar has to be thicker than the disk. We find that NGC 7331, even though it is a fairly early-type spiral, does not have a conventional, co-rotating bulge. The fact that the inner component is retrograde makes us believe that it was formed from infalling material, in either stellar or gaseous form (e.g. Balcells \& Quinn 1990). Another possibility
\end{abstract}


however is that the structure has been there since the formation of the galaxy. In this case it will be a challenge to explain the large change in orientation of the angular momentum when going outward radially.

Subject headings: galaxies: individual (NGC 7331) — galaxies: kinematics and dynamics — galaxies: spiral — galaxies: structure — galaxies: formation

\section{INTRODUCTION}

In the last decade many elliptical galaxies have been found which display complicated multiple-component structure in their kinematics and, in some cases, also in their photometry. This structure has been interpreted as being due to mergers (e.g. Balcells \& Quinn 1990), to components which were formed later from processed gas (Bender \& Surma 1992), or as triaxial systems in projection (Statler 1991). Sometimes these multiple components give rise to disky isophotes, so that they can be found using photometry. However in general they are much better seen in the analysis of the line-of-sight velocity distribution (LOSVDs). Examples of galaxies with multiple components can be found in e.g. Franx \& Illingworth (1988), Rix \& White (1992) and Bender et al. (1994). Typically they consist of a bright, pressure-supported body, and a fainter, fast-rotating central disk, although this is not always the case (e.g. Balcells \& Carter 1993).

The kinematics of stars in spiral galaxies and lenticulars has been much less studied. Bulges are generally thought to be supported by rotation (Kormendy 1993) and no non-rotating bulge has been found up to now, except maybe for the very small bulge of NGC 4550 (Franx 1993). They rotate in the same direction as the disks, although in general they are also partly pressure-supported. Recently however, a number of cases has been found in which a fraction of the stars in the disk rotates in a retrograde way. These include NGC 4550 (Rubin et al. 1992, Rix et al. 1992), NGC 7217 (Merrifield \& Kuijken 1994) and NGC 3593 (Bertola et al. 1996). These counter-rotating disks are thought to have originated from infalling gas, accreted in retrograde orbits in a plane close to the equatorial plane (Merrifield \& Kuijken 1994).

In this paper we present the discovery of a different type of galaxy: one in which the bulge rotates retrograde to the disk. We have found this by analyzing LOSVDs along the major axis of the large, nearby Sb (Sandage \& Tammann 1981) galaxy NGC 7331. Here two components are seen, of which the slow, counter-rotating one corresponds to the boxy 
bulge of this galaxy. Explaining the formation of a system like this will be a challenge to galaxy formation theories. Section 2 of this paper gives details about the observations. In Sections 3 and 4 we describe our analysis of morphology and the LOSVDs. The discussion and conclusions are given in Section 5 .

\section{OBSERVATIONS AND DATA REDUCTION}

We obtained long-slit spectra with the ISIS spectrograph on the $4.2 \mathrm{~m}$ WHT at La Palma (August 1992) along the major axis (p.a. 167', Bosma 1981) of NGC 7331 . The slit was centered on the apparent optical nucleus, with a projected slit width of $1^{\prime \prime}$, slightly undersampling the $1^{\prime \prime}$ seeing. The spectral dispersion was 2.1 pixels of $25 \mathrm{~km} \mathrm{~s}^{-1}$ pixel $^{-1}$ each. The spectrum, of $1800 \mathrm{~s}$, was taken in the red arm of ISIS, with the wavelength centered at $8700 \AA$ to include the CaII IR triplet $(8494,8542,8662 \AA)$. The bias subtraction, flat-fielding and wavelength calibration of the data were carried out using FIGARO. Sky lines were subtracted using template spectra from the edges of the slit. As a stellar template star we observed the K0 giant HR 5631. To confirm and strengthen our results we have also analyzed a spectrum from the La Palma Archive, of NGC 7331 along p.a. $172^{\circ}$. These data were taken with the same instrumental setting and exposure time in September, 1991, by D. Carter and M. Balcells. Their seeing FWHM was $1^{\prime \prime}$ and their spectral resolution was 2.5 pixels FWHM. From the same observing run we used the G8 giant HR 7753 as a template.

To study the morphology, we took images in the Cousins $I$-band and the infrared $K$-band. The $I$-band image was taken in June 1990 at the INT at La Palma with the $2.5 \mathrm{~m}$ INT and a $590 \times 400 \mathrm{EEV} \mathrm{CCD.} \mathrm{The} \mathrm{pixel} \mathrm{size} \mathrm{was} 0.549^{\prime \prime}$, with a seeing of $\approx 1.2^{\prime \prime}$. The $K$-band data were obtained at UKIRT in June 1994 using IRCAM3 with a $256 \times 256 \mathrm{InSb}$ detector. A mosaic was made to have a total field of $80^{\prime \prime} \times 80^{\prime \prime}$, with pixels of $0.291^{\prime \prime}$. The data were taken under photometric conditions with a seeing of $\approx 0.9^{\prime \prime}$. Details of the data reduction can be found for the optical data in Balcells \& Peletier (1994) and for the infrared data in Peletier \& Balcells (in preparation).

\section{THE STRUCTURE OF NGC 7331}

To determine the structure of the central regions of NGC 7331 we fitted ellipses to the images in the $K$ - and $I$-band using Galphot (see Jørgensen et al. 1992). Fig. 1 shows the 
surface brightness profiles in $I$ and $K$ as a function of major axis radius. There are some minor differences in the inner $60^{\prime \prime}$ between both profiles, mostly due to extinction by dust. Because of the smaller size of the $K$-band image it was not possible to extend the fit as far as in the $I$-band. Consequently the slope of the profile in $K$ in its outer parts is much steeper than the slope of the profile in $I$ at large $I$-band radii. If one tries to decompose the galaxy into an exponential disk and a $\mathrm{r}^{\frac{1}{4}}$ bulge, only based on the surface brightness profile, one will get a much larger bulge in $I$ than in $K$ (see Fig. 1). In galaxies that are not face-on however, one can also use the information available in the axis ratio distribution, and in this way try to isolate a flat disk and a rounder bulge (Kent 1985). Applying Kent's decomposition method for this galaxy of inclination $75^{\circ}$ we have obtain in both bands the fit that was obtained in $K$ using the previous method. The reason for this is that the ellipticity in this galaxy only changes up to $r=\sim 10^{\prime \prime}$, so that the only possible solution can be a small bulge and a large inclined disk. In Fig. 2 we have plotted ellipticity, major axis position angle and boxyness $\mathrm{C} 4$ as a function of major axis radius.

It seems that the morphology shows three large components: the central bulge, with effective radius of $\sim 10^{\prime \prime}(K)$, and then two flat components: an intermediate component with scale length of $\sim 25^{\prime \prime}$, and from $60^{\prime \prime}$ onwards the large outer disk.

Looking in more detail at the position angle and $\mathrm{C} 4$ major axis profiles we see in the inner $5^{\prime \prime}$ a boxy structure ( $\mathrm{C} 4=-0.017$ at $2.5^{\prime \prime}$ ) and a position angle twist of $10^{\circ}$ between $2.5^{\prime \prime}$ and $10^{\prime \prime}$. This component is entirely part of the bulge. Between $5^{\prime \prime}$ up to $15^{\prime \prime}$ the morphology is suddenly very disky, with $\mathrm{C} 4$ values $\sim+0.04$ which are much larger than the normal values for disky elliptical galaxies (e.g. Bender et al. 1988). Beyond 15", C4 depends on passband, and is probably severely affected by extinction. At larger radii it stabilizes to a value of $\sim+0.02$. No major changes of position angle and ellipticity are seen beyond $10^{\prime \prime}$.

\section{THE LINE-OF-SIGHT VELOCITY DISTRIBUTION IN NGC 7331}

To obtain the LOSVDs, we compared the shift and Doppler broadening of the CaII IR triplet region with a standard reference stellar spectrum (K0III) recorded the same night and with the same instrumental configuration as for the galaxy. We used an extension of the unresolved Gaussian decomposition technique developed by Kuijken \& Merrifield (1993). The basic difference between their and our algorithm is that we perform a two-dimensional fit to the long-slit spectra by using unresolved Gaussian components with dispersion 2 pixels and separation 3 pixels in both spatial and spectral direction. In general the results of both algorithms are the same, but ours should be more robust to spurious features in 
regions with low signal to noise ratios. A detailed description of the algorithm will be given in a separate paper (Gutiérrez \& Prada, in preparation). In Plate 1, a surface plot of the LOSVDs along the major axis of NGC 7331 is shown. The LOSVDs in the inner $4^{\prime \prime}$ are nearly symmetric (see Fig. 3, top panel). Between $4^{\prime \prime}$ and $7^{\prime \prime}$ on both sides of the nucleus a strong asymmetry is evident towards the systemic velocity (see Fig. 3, middle panel) of the galaxy. Between $7^{\prime \prime}$ and $15^{\prime \prime}$ the LOSVDs show two separate peaks, with the fainter one crossing the systemic velocity (see Fig. 3, bottom panel). Further out, the fainter peak disappears and the LOSVDs are again defined by a single component. The archive spectrum was analyzed in the same way, and the same features were found. We have fitted one, and where possible two Gaussians to the LOSVDs, and display the kinematic parameters (recession velocity, velocity dispersion and flux) as a function of major axis radius in Fig. 4. Because of the way our algorithm works the individual points are slightly correlated; the algorithm effectively smoothes the data in this figure in the spatial direction with a Gaussian of FWHM 1.6". We find that between $5^{\prime \prime}$ and $15^{\prime \prime}$ on both sides of the nucleus a fraction of the stars rotates slowly and retrograde to the rest of the galaxy. In the inset of Fig. 1 we have plotted the relative intensity of both components, obtained from the LOSVDs. The profile of the fainter component agrees well with that of the bulge, as obtained from the photometric decomposition in K. In the inner $5^{\prime \prime}$, since an unambiguous decomposition of the line profiles was not possible due to lack of resolution, we have fixed the velocity of the bulge component to the velocity of the dashed line in Fig. 4, as well as the intensity ratio of both components, which we assumed to be the ratio of the components from the photometric decomposition. It was only possible to obtain reasonable decompositions if the dispersion of the low-velocity component was taken to be $110 \mathrm{~km} / \mathrm{s}$ or larger. For that reason its dispersion was fixed at $110 \mathrm{~km} / \mathrm{s}$. The velocities obtained for the disk are plotted in the upper panel of Fig. 4 as filled circles.

\section{DISCUSSION AND CONCLUSIONS}

To summarize the observations: the inner parts of the galaxy consist of a boxy component, dominating the inner $5^{\prime \prime}$. It shows position angle twisting, rotates retrograde to the rest of the galaxy, and is rounder. Outside $5^{\prime \prime}$ the disk dominates. This component is much colder, is probably flat, and responsible for the disky isophotes. At appr. $r=100^{\prime \prime}$ the surface brightness profile becomes shallower.

The boxyness and position angle twist make it very likely that we are seeing a triaxial object counter-rotating to the main body of the galaxy. It could be that we have a 
configuration like in NGC 4736 (Möllenhoff et al. 1995), where the bar is oriented end-on towards us, or alternatively, that the galaxy has no bar, and that we are seeing a triaxial bulge. The steep rise in the surface brightness profile and the low ellipticity show that it can't be a classical, flat bar with a Freeman-type profile (Freeman 1966). From this data we cannot distinguish between these two alternatives any further. Whatever the case, the galaxy does not contain a large, co-rotating bulge; if it has a bar a small bulge, as in NGC 4736, can be hidden.

It is likely that the origin of the central component is external. Evidence for this would be the fact that it is retrograde, and maybe the boxyness. Simulations for elliptical galaxies have shown that it is possible to create a central, counter-rotating body as a result of a stellar merger (Balcells \& Quinn 1990). Another possibility is that the central component is formed as a result of an instability in the disk of counter-rotating stars, like the one of NGC 7217 (Merrifield \& Kuijken 1994). In that case however the counter-rotating disk must have accreted from outside first. A third possibility might be that the counter-rotating stars were formed at the formation epoch together with the rest of the galaxy. Up to now no models have been proposed where two counter-rotating components were formed from one protogalactic cloud, and where the angular momentum changes so drastically as a function of radius. We might observe here something similar to a counter-rotating bar within a bar, for which Friedli (1996) has shown that it can be very longlived.

Further evidence for a triaxial potential in the center of this galaxy comes from the $\mathrm{H} \alpha$ velocity field by Marcelin et al. (1994). They find the typical Z-shape in the isovelocity contours, characteristic of non-circular potentials. The galaxy shows some low-level activity (Ho et al. 1995). On larger scales, the motion of the gas is quite regular (von Linden et al. 1996, Begeman 1987).

We now ask ourselves how unique this galaxy is. Studies using e.g. the Fourier quotient method (Tonry \& Davis 1979) generally find that the bulge is dynamically supported by rotation, and rotates in the same direction as the disk (Kormendy 1993). If however the bulge to disk ratio diminishes, it becomes difficult to find any slow-rotating component in this way. To show this, we give the $\mathrm{h} 3$ and $\mathrm{h} 4$ components, as defined by van der Marel \& Franx (1993), for NGC 7331 in Fig. 3. They are similar to h3 and h4 profiles of many elliptical galaxies (e.g. Bender et al. 1994). Since however a somewhat fainter, co-rotating component could give the same $\mathrm{h} 3$ and $\mathrm{h} 4$ as we are seeing here, we need to investigate the line profiles in more detail. By now, there are several spiral galaxies and S0's for which the line profiles have been studied, There are many cases with a hot bulge and a cold disk rotating in the same direction (e.g. NGC 3115 (van der Marel et al. 1994), NGC 4736 (Möllenhoff et al. 1995), NGC 4594 (Wagner et al. 1989)). But as far as we know, no galaxy 
has been found where the central and outer component rotate opposite to each other. We conclude that NGC 7331 is special in this respect.

We would like to end advancing some speculations. First, we find here a very boxy, slowly rotating inner component, and a large, fast rotating disk. Many giant elliptical galaxies on the other hand have a boxy, even slower rotating, main body, and a small, rotating disk. Apart from the relative scales, the similarity of both systems is striking, and has to imply that the formation scenario of spirals like NGC 7331 and ellipticals cannot be too different. And secondly, we see a discontinuity in the slope of the surface brightness profile at $r=100^{\prime \prime}$. Since this galaxy is of type $\mathrm{Sb}$, it is rather peculiar that it doesn't have a larger, co-rotating bulge. Could it be that the component that dominates the light between $5^{\prime \prime}$ and $100^{\prime \prime}$ was the previous bulge? It however is flat, rotates fast, and is disky, but according to Kormendy (1993) many late type spirals have 'bulges' that look like this. This question obviously is still open, and needs much more investigation.

This research is based on observations obtained at the WHT and INT, operated by the RGO at the Observatorio del Roque de los Muchachos at La Palma of the IAC. We thank E. Simonneau, E. Perez, M. Balcells, K. Kuijken, D. Fisher, M. Franx, R. Bottema and R. Sancisi for helpful discussions. The spectroscopic observations were performed on a service night (August 1992) by Dr. R. Clegg. Part of the observations are based on the La Palma Archive of the Royal Greenwich Observatory. We thank the referee for very valuable suggestions, and T. Mahoney for his help with the text.

\section{REFERENCES}

Balcells, M. \& Quinn, P.J., 1990, ApJ, 361, 381

Balcells, M. \& Carter, D. 1993, A\&A, 279, 276

Balcells, M. \& Peletier, R.F., 1994, AJ, 107, 135

Begeman, K., 1987, PhD thesis, University of Groningen

Bender, R., Döbereiner,S. \& Möllenhof,C., 1988, A\&AS, 74, 385

Bender, R. \& Surma, P., 1992, A\&A, 258, 250

Bender, R.,, Saglia, R.P. \& Gerhard, O.E., 1994, MNRAS 269, 785

Bertola, F., Cinzano, P., Corsini, E.M., Pizzella, A., Persic, M. \& Salucci, P., 1996, ApJ, 458, L67

Bosma, A., 1981, AJ, 86, 1791 
Franx, M., 1993, in Galactic Bulges, ed. H. Dejonghe \& H.J. Habing, Kluwer, Dordrecht, p. 243

Franx, M. \& Illingworth, G., 1988, ApJ, 327, L55

Freeman, K.C., 1966, MNRAS, 133, 47

Friedli, D., 1996, A\&A, in press

Ho, L.C., Filippenko, A.V. \& Sargent, W.L., 1995, ApJS, 98, 477

Jørgensen, I., Franx, M. \& Kjaergaard, P., 1992, A\&A S, 95, 489

Kent, S.M., 1985, ApJS, 59, 115

Kormendy, J., 1993, in Galactic Bulges, ed. H. Dejonghe \& H.J. Habing, Kluwer, Dordrecht, p. 209

Kuijken, K. \& Merrifield, M.R., 1993, MNRAS, 264, 712

Marcelin, M., Petrosian, A.R., Amram, P. \& Boulesteix, J., A\&A, 282, 363

Merrifield, M.R. \& Kuijken, K., 1994, ApJ, 432, 575

Möllenhoff, C., Matthias, M. \& Gerhard, O.E., 1995, A\&A, 301, 359

Rix, H.-W. \& White, S.D.M., 1992, MNRAS, 254, 389

Rix, H-W., Franx, M., Fisher, D. \& Illingworth, G., 1992, ApJ, 400, L5

Rubin, V., Graham, J. \& Kenney, J., 1992, ApJ, 394, L9

Sandage, A. \& Tammann, G.A., 1981, A Revised Shapley-Ames Catalog of Bright Galaxies. (Carnegie Institution of Washington Pub. No. 635)

Statler, T.S., 1991, AJ, 102, 882

Tonry, J. \& Davis, M., 1979, AJ, 84, 1511

van der Marel, R.P. \& Franx, M., 1993, ApJ, 407, 525

van der Marel, R.P., Rix, H.-W., Carter, D., Franx, M., White, S.D.M. \& de Zeeuw, P.T., 1994, MNRAS, 268, 521

von Linden, S., Reuter, H.-P., Heidt, J., Wielebinski, R. \& Pohl, M., 1996, A\&A, in press Wagner, S.J., Bender, R. \& Dettmar, R.J., 1989, A\&A, 215, 243 


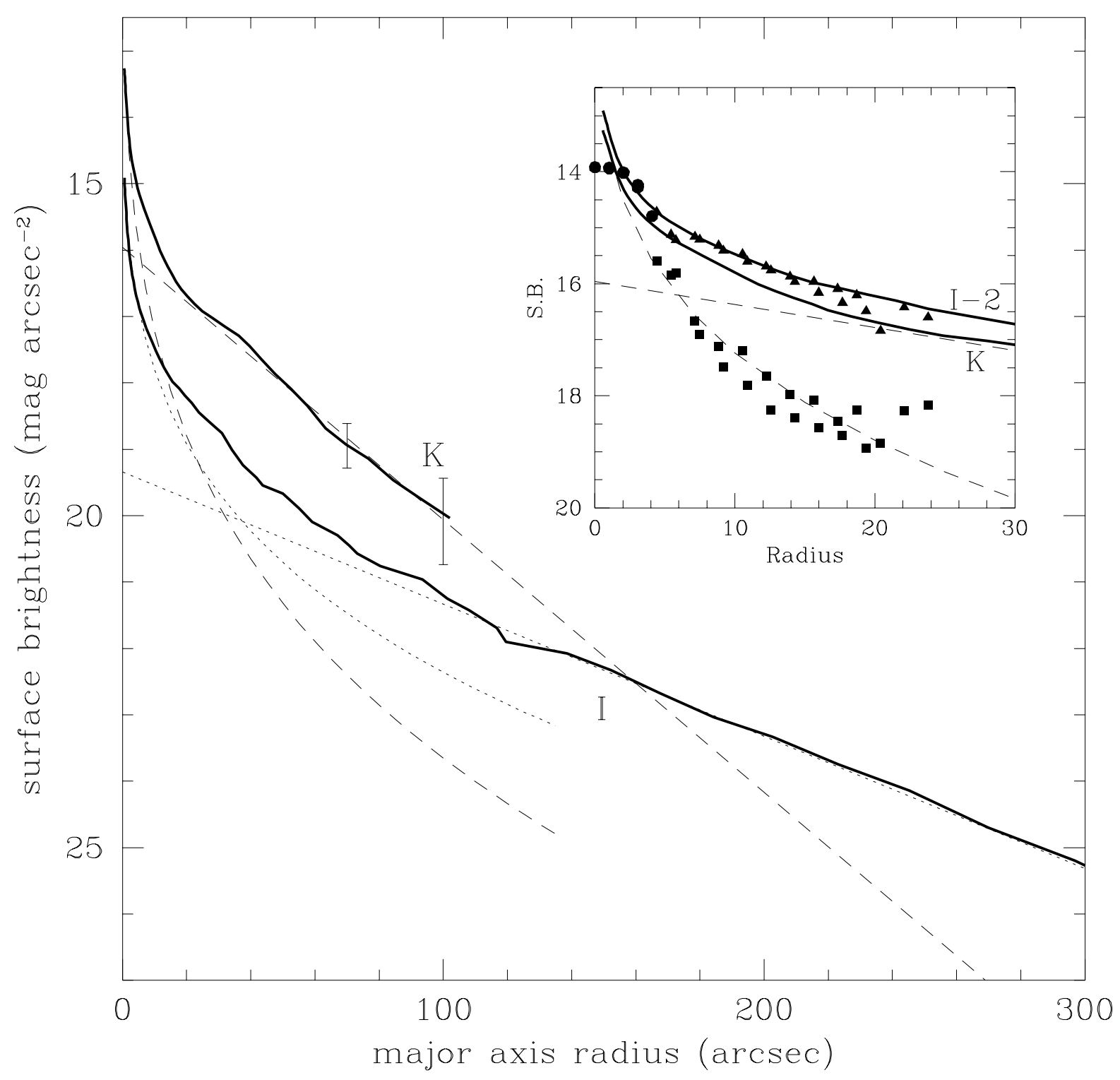

Fig. 1. - Plot of the major axis surface brightness profile of NGC 7331 in $I$ and $K$ (solid lines). Both profiles has been decomposed into an exponential disk and an spheroidal $\mathrm{r}^{1 / 4}$ component (dashed and dotted lines for the $K$ and $I$-band respectively). The fit parameters are also shown. Also plotted are the one-sigma error-bars due to uncertainties in the determination of the sky background. ( $1^{\prime \prime} \sim 72 \mathrm{pc}$ for a distance of $14.9 \mathrm{Mpc}$, see Begeman 1987). In the inset we have enlarged the central regions. Here also plotted are the fluxes of the high (triangles) and low-velocity (squares) components, from the decomposition of the line profiles, shifted by an arbitrary constant. The I-band profile (minus 2 magnitudes) is also shown here. 


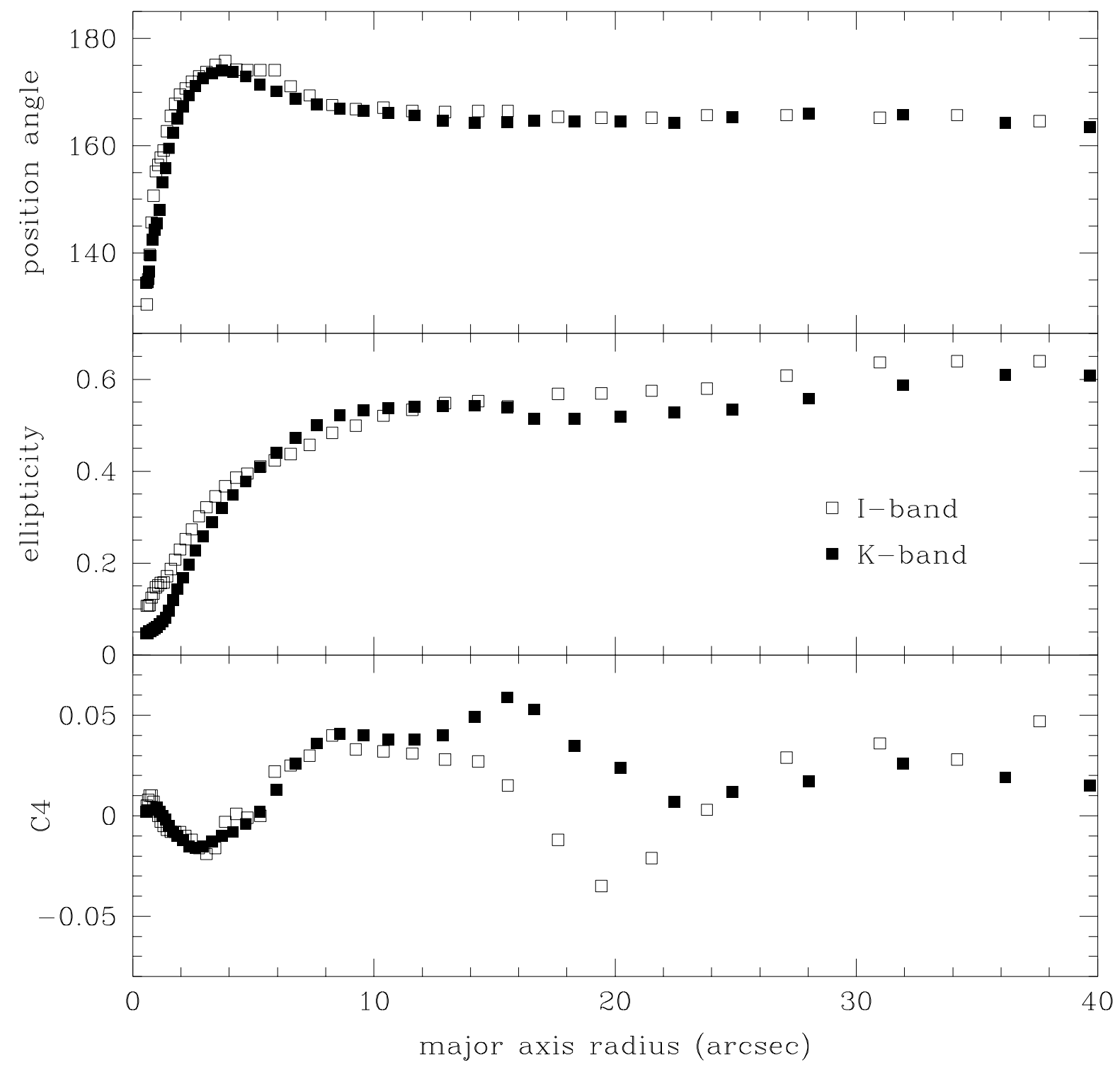

Fig. 2.- Isophotal analysis of the $I$ - and $K$-band surface photometry of NGC 7331. Top panel: the major axis position angle profile; middle panel: the ellipticity profile; and the bottom panel: the profile of the $\mathrm{C} 4$ Fourier coefficient indicating deviations from elliptical shape. 

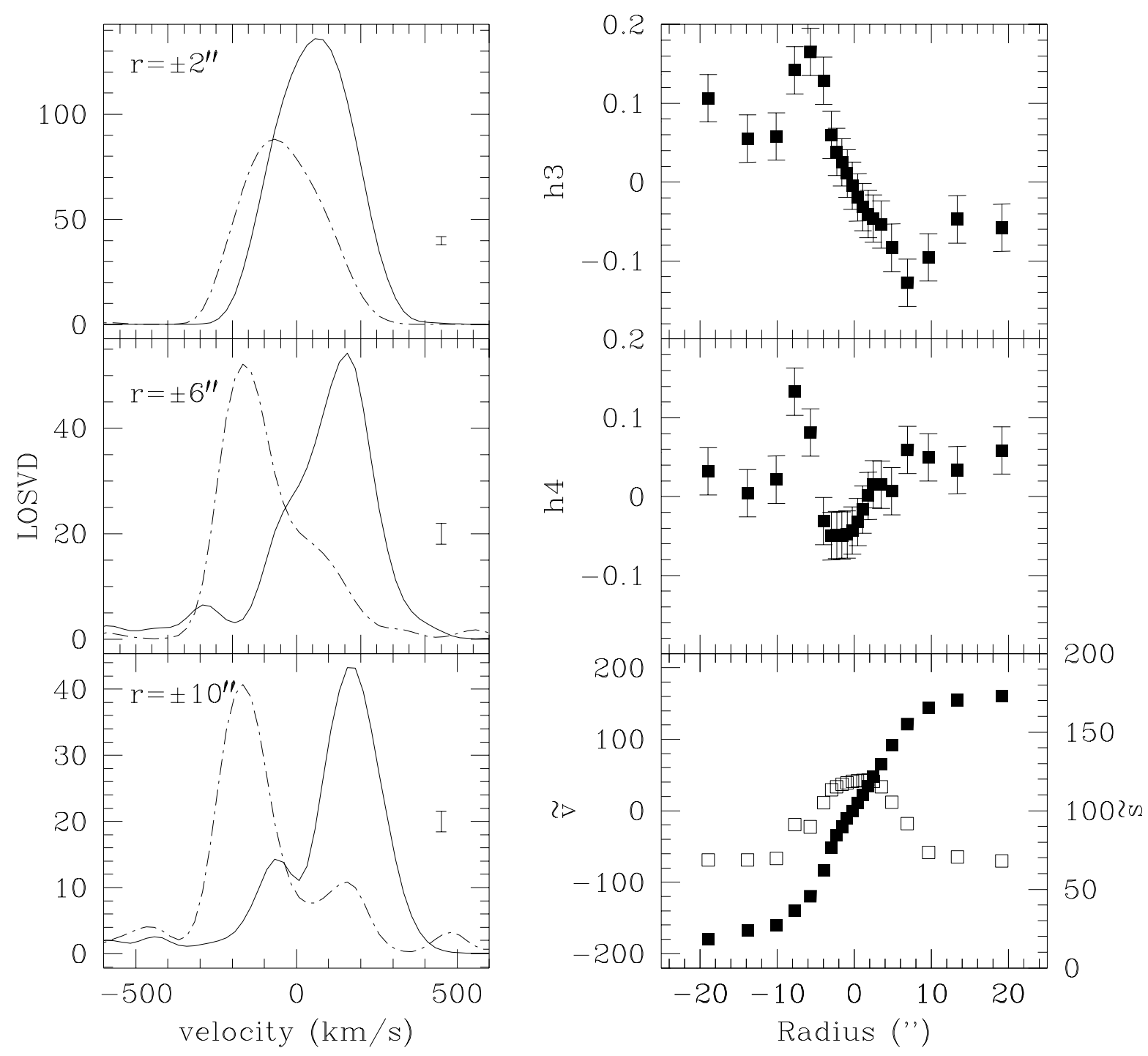

Fig. 3.- On the left three LOSVDs along the major axis of NGC 7331 at different distances from the center. Dot-dashed line represent the NW side (blueshift) and full line the SE side (redshift). Typical error-bars are shown. On the right we give the profiles of h3, h4, v (filled symbols) and $\tilde{\sigma}$ according to the definition of van der Marel \& Franx (1993). 


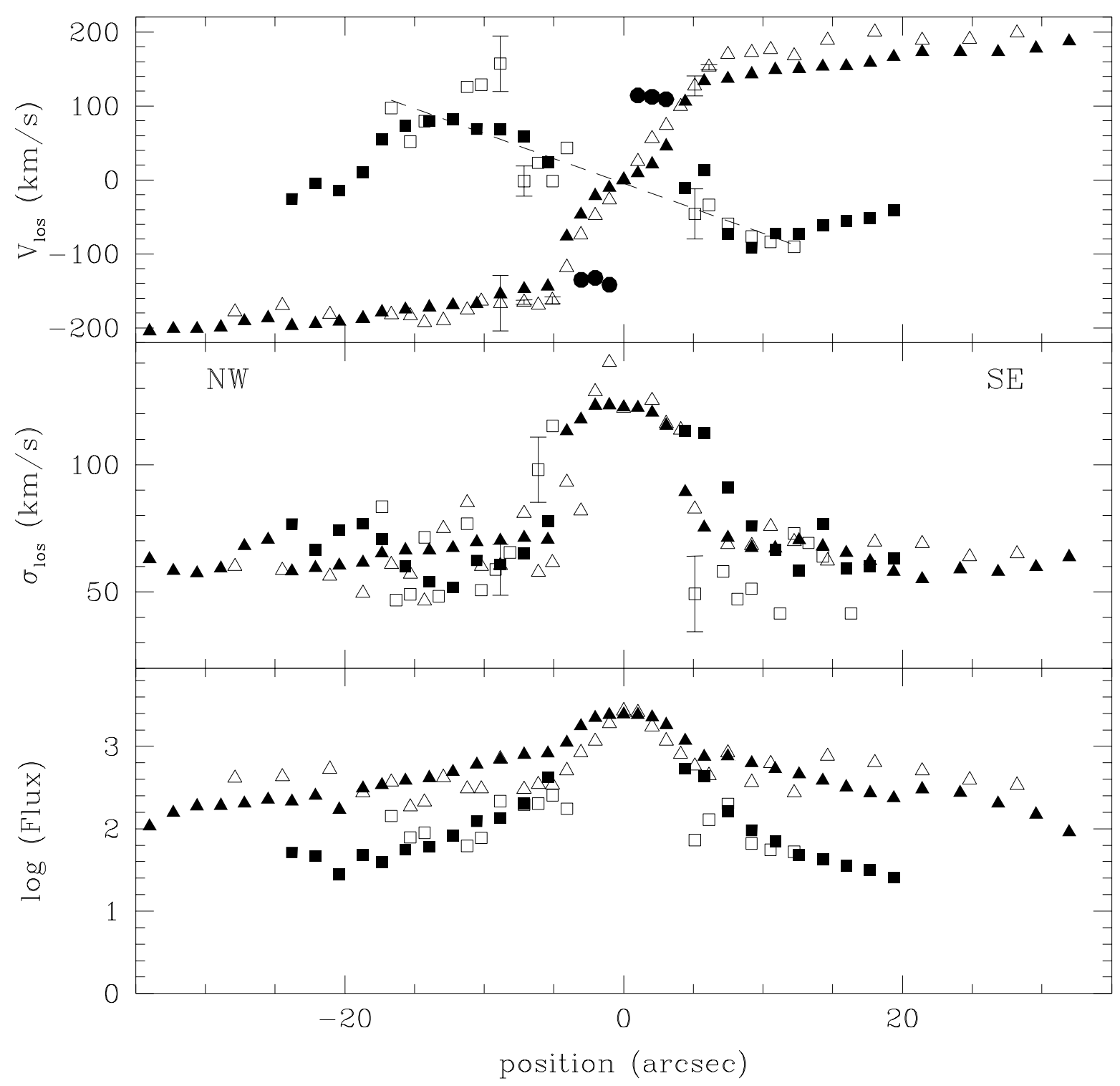

Fig. 4.- Kinematic parameters for fitting two Gaussians to the LOSVDs along the major axis of NGC 7331. Open and filled symbols correspond to the independent spectra at p.a. $167^{\circ}$ and p.a. $172^{\circ}$ respectively (our observations and those from the archive). Triangles and square symbols refer to the brighter and fainter components present in the LOSVDs. The errors in the fit to the LOSVDs are comparable in general to the size of the symbols, larger errors are indicated. The dashed line in the top panel is a model for the bulge component. In the inner parts, where no unambiguous decomposition was possible, we have fixed the velocity and dispersion of this component, as well as the flux ratio (see text). The highvelocity component that is obtained in this way is indicated with filled circles. 


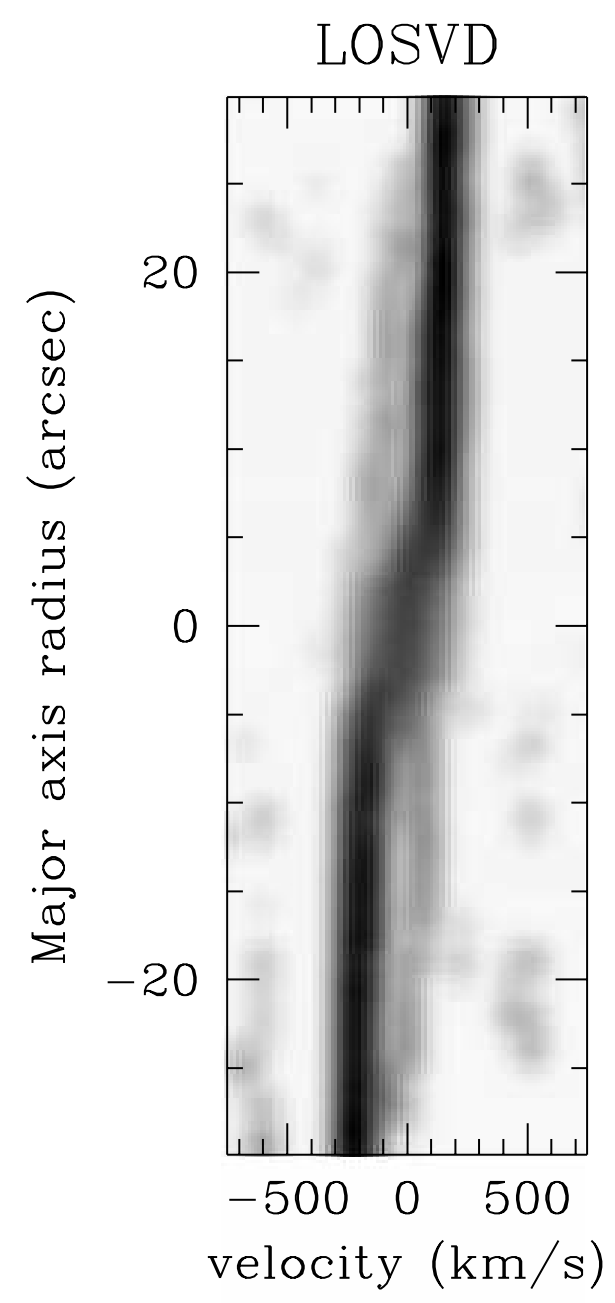

Fig. 1.- Plate 1: Greyscale plot of the normalized stellar line-of-sight velocity distribution along the major axis of NGC 7331, where for presentation purposes the data in the spatial direction has been smoothed with a Gaussian with a FWHM of $4^{\prime \prime}$. 